\title{
Gallbladder Metastasis from Renal Cell Carcinoma Mimicking Acute ChOLECYSTITIS
}

\author{
M. Sand ${ }^{1}$, F.-G. Bechara' 2 J. Kopp ${ }^{3}$, N. Krins 3 , D. Behringer ${ }^{4}$, B. Mann ${ }^{1}$ \\ ${ }^{1}$ Department of General and Visceral Surgery, Augusta Krankenanstalt, Academic Teaching Hospital, \\ 2Department of Dermatology and Allergology, St. Josef Hospital, Ruhr University Bochum, Germany \\ ${ }^{3}$ Department of Radiology, Augusta Krankenanstalt, Academic Teaching Hospital, \\ ${ }^{4}$ Department of Oncology, Augusta Krankenanstalt, Academic Teaching Hospital,
} Ruhr University Bochum, Germany

\begin{abstract}
Renal cell carcinoma constitutes about $3 \%$ of adult malignancies. It has a high metastatic potential associated with synchronous or metachronous metastatic disease. Further, it is known to metastasize mainly to the lung, bone, brain, liver, or adrenal glands. In very rare cases it can metastasize to the gallbladder mimicking acute cholecystitis on clinical exam. In this case we present a patient who developed a gallbladder metastasis five years after a renal cell carcinoma mimicking acute cholecystitis.
\end{abstract}

\section{CASE}

In November, 2007, a 48-year-old woman presented in our clinic with a four-day history of colicky pain in the right upper quadrant radiating to the back. Additionally she felt pain in her right scapular region. She complained of nausea and vomiting. She had a history of nephrectomy and adrenalectomy for right sided renal cell carcinoma (RCC) in 2002. Since then all yearly follow ups consisting of abdominal sonography and CTscan were without pathological findings.

Physical examination revealed axillary temperature of $37.8 \mathrm{C}$, regular heart beat ( 84 beats/min) without heart murmur, and normal breath sounds. Examination of the abdomen revealed tenderness in the right upper quadrant, positive Murphy's sign and unremarkable bowel sounds. Laboratory analysis showed a slight thrombopenia with $139.00 / \mathrm{NL}$ and haemoglobin of $10.9 \mathrm{~g} / \mathrm{dl}$. All other laboratory values were within normal limits, there was no cholestasis and no laboratory signs of infection.

Ultrasound showed a wall thickness of the gallbladder $>6 \mathrm{~mm}$, consistent with an enlargement of the organ and hyperplasia of its wall. Further sludge in the lumen and a light retention of effusion around the gallbladder was noted (Fig. 1). All other organs showed no pathological finding in the pre-operative ultrasound. Because the acute symptomatic dictated an operation a pre-operative CT-scan was not performed. The patient underwent cholecystectomy which was planned laparoscopic but extended to conventional cholecystectomy because of multiple post-nephrectomy adhesions in 2002.

Histopathologic examination revealed a metastatic tumour which appeared macroscopically as a $2.5 \mathrm{x} 2 \mathrm{x}$ $1.5 \mathrm{~cm}$ polyp at the neck of the gallbladder (GB). Histopathology showed clear cells of renal carcinoma with delicate vascular stroma as already seen in the primary tumour of the nephrectomy specimen (Fig. 2). An abdominal CT-scan showed additional space occupying lesions in the pancreas and the left kidney. As this was interpreted as advanced metastatic renal cell carcinoma a palliative therapy with Sorafenib (Nexavar ${ }^{\circledR}$ tablets 2 × $200 \mathrm{mg} / \mathrm{d}$, Bayer Pharmaceuticals Corp.) was initiated. Although Sunitinib would be preferred by most oncologists as first line treatment Sorafenib was chosen because of the favorable profile of side effects, especially with respect to fatigue [1]. When the patient was last seen in January 2008 she was in good condition.

\section{DiscusSION}

Renal Cell Carcinoma (RCC) is a rare tumour accounting for $3 \%$ of all adult malignancies. It has a high

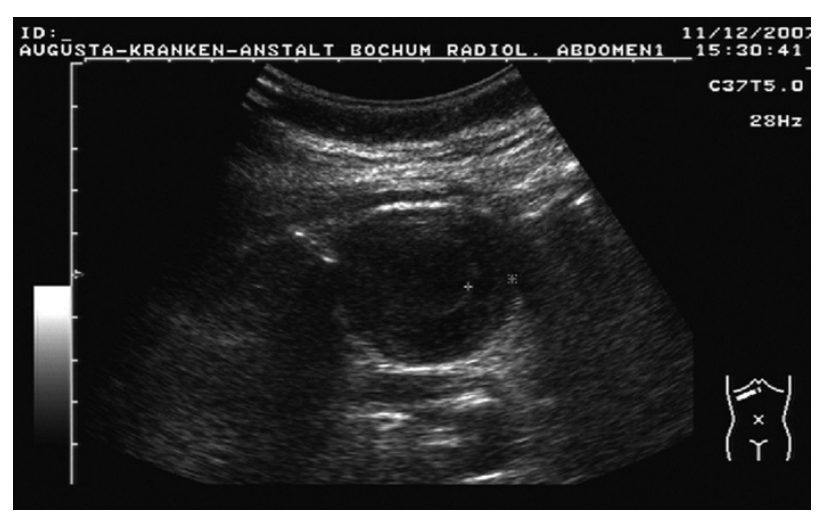

Fig. 1. Ultrasound of the gallbladder consistent with cholecystitis. Additionally sludge in the lumen (blue marks) and a light retention of effusion around the gallbladder was noted. 


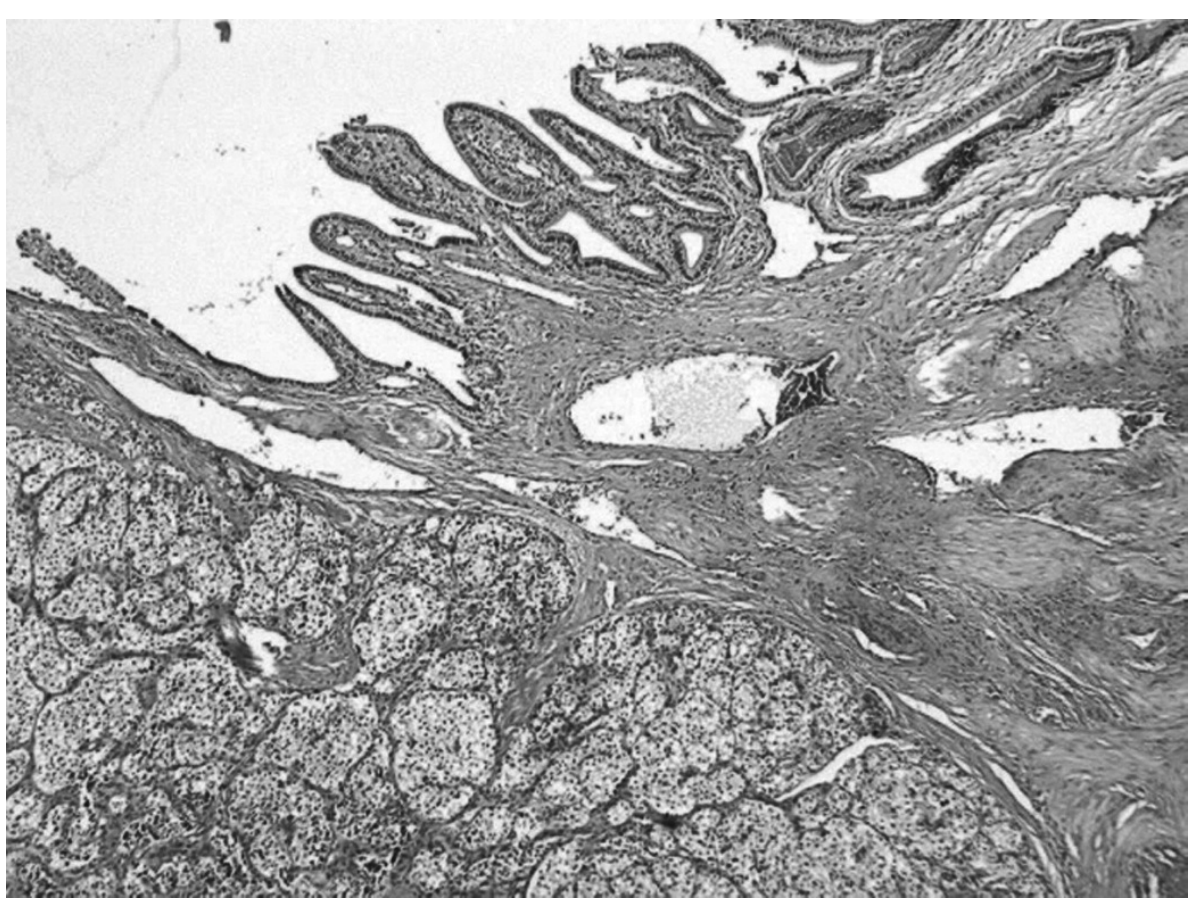

Fig. 2. Histopathological examination showed clear cells of renal carcinoma (left) and regular gall-bladder epithelium (right) (haematoxylin and eosin stain; x 40 magnification). metastatic potential with $25-57 \%$ metastatic disease at time of initial presentation [2]. Metastatic RCC has a poor prognosis with 1 -year survival of less than $50 \%$, 5 year suvival of $5-30 \%$, and 10 year survival of $0-5 \%$ [3]. RCC is known to metastasize mainly to the lung, bone, brain, liver, or adrenal glands [4]. RCC metastases to the gallbladder are very unusual, only a few case reports are available in the literature of surgically resected metastasis documenting this rare localization. Diagnosis of gall bladder metastasis was more often metachronous than synchronous. In the reviewed cases metachronous tumours were detected from 3 months to 27 years after initial diagnosis $[5,6]$. However the vast majority is detected at autopsy [7]. In a study by Bennington et al. the incidence of gallbladder metastasis of RCC was reported to be $0.57 \%$ ( 3 out of 523 autopsies) [8]. In another study Weiss et al. report $0.58 \%$ (4 out of 687 autopsies) [9].

Gallbladder metastasis have been reported from other primary tumour, melanoma being the most frequent followed by lung, oesophagus, pancreas and colon carcinoma [10]. Metastasis from extraperitoneal organs such as the kidney, breast, skin and lung are described to primarily follow a hematogenous route [11]. Willis reported that hematogenous metastasis to the gallbladder initially begin with small flat submucosal nodules which then grow as pedunculated tumors, being rarely greater then several mililimeter in size resembling primary GB carcinoma $[12,13]$.

As in our patient, metastatic RCC to the gallbladder may produce symptoms mimicking cholecystitis which is an uncommon and unusual clinical presentation of metastatic disease. For primary gallbladder malignancies in general and especially for secondary gallbladder metastasis, conventional diagnostic methods including history, physical examination and sonography are nonspecific. Although Color Doppler sonography and CT findings have been described for metastatic renal cell carcinoma to the gallbladder they are still unspecific as metastasis show a broad variety of presentation [6]. Hypervascularity has been reported to be a sign useful in distinguishing metastatic GB tumor of RCC from primary GB tumor which is described to be not so hypervascular. Nevertheless Doppler sonography was not performed in our patient.

\section{CONCLUSION}

As a conclusion from this case, patients with a history of RCC (even after several years of tumour free disease) and pain in the right upper quadrant deserve a high index of suspicion regarding gall bladder metastasis which should be considered as a rare but possible cause. An intraoperative examination of gallbladder specimens and histopathologic examination should be mandatory. Additionally, a pre-operative CT-scan may be considered which potentially alters further therapy.

Acknowledgements: The written consent was obtained from the patient. We thank Professor Dr. Stathis Philippou, Chief of the Department of Pathology, Augusta Krankenanstalt, Academic Teaching Hospital of the Ruhr-University Bochum, for providing the histologic image.

\section{REFERENCES}

1. Schrader AJ, Hofmann R. Metastatic renal cell carcinoma: recent advances and current therapeutic options. Anticancer Drugs. 2008 Mar;19(3):235-45. Review.

2. Park JS, Chae YS, Hong SJ, Shin DH, Choi JS, Kim BR. Metastatic renal cell carcinoma of the gallbladder. Yonsei Med J. 2003 Apr 30;44(2):355-8.

3. Motzer RJ, Russo P. Systemic therapy for renal cell carcinoma. J Urol. 2000 Feb;163(2):408-17. Review.

4. Maldazys JD, deKernion JB. Prognostic factors in metastatic renal cell carcinoma. J Urol. 1986 Aug;136(2): 376-9.

5. Fullarton GM, Burgoyne M. Gallbladder and pancreatic metastases from bilateral renal carcinoma presenting with hematobilia and anemia. Urology. 1991 Aug;38(2):184-6. 
6. Furukawa H, Mizuguchi Y, Kanai Y, Mukai K. Metastatic renal cell carcinoma to the gallbladder: color Doppler sonography and CT findings. AJR Am J Roentgenol. 1997 Nov;169(5):1466-7.

7. Hellsten S, Berge T, Wehlin L.Unrecognized renal cell carcinoma. Clinical and diagnostic aspects. Scand J Urol Nephrol. 1981;15(3):269-72.

8. Bennington JL, Kradjan RM (1967) Distribution of metastases from renal carcinoma. In: Renal carcinoma. Saunders, Philadelphia, pp 156-170.

9. Weiss L, Harlos JP, Torhorst J, Gunthard B, Hartveit F, Svendsen E, Huang WL, Grundmann E, Eder M, Zwicknagl $\mathrm{M}$, et al. Metastatic patterns of renal carcinoma: an analysis of 687 necropsies. J Cancer Res Clin Oncol. 1988;114(6):605-12.

10. Coskun F, Cetinkaya M, Cengis O, Adsan O, Kualcoglu S, Eroglu A. Metastatic carcinoma of the gallbladder due to renal cell carcinoma in the ectopic kidney. Acta Chir Belg. 1995 Jan-Feb;95(1):56-8.

11. Murguia E, Quiroga D, Canteros G, Sanmartino C, Barreiro M, Herrera J. Gallbladder metastases from ductal papillary carcinoma of the breast. J Hepatobiliary Pancreat Surg. 2006;13(6):591-3.
12. Aoki T, Inoue K, Tsuchida A, Aoki T, Kasuya K, Kitamura K, Koyanagi Y, Shimizu T. Gallbladder metastasis of renal cell carcinoma: report of two cases. Surg Today. 2002;32(1):89-92.

13. Willis RA, The spread of tumours in the human body. Butterworth, London, pp 218-219

Received: March 13, 2008 / Accepted: July 18, 2008

Address for correspondence:

Dr. Michael Sand

Department of General and Visceral Surgery

Augusta Krankenanstalt

Academic Teaching Hospital of the Ruhr-University Bochum,

Bergstr. 26

44791 Bochum

Germany

E-mail: michael.sand@ruhr-uni-bochum.de 